\title{
Reduction of the flash-lag effect in terms of active observation
}

\author{
МАKOTO ICHIKAWA \\ Chiba University, Chiba, Japan \\ AND \\ Yuko Masakura \\ Tokyo Polytechnic University, Kanagawa, Japan
}

\begin{abstract}
In the present study, we investigated how observers' control of stimulus change affects temporal and spatial aspects of visual perception. We compared the illusory flash-lag effects for automatic movement of the stimulus with stimulus movement that was controlled by the observers' active manipulation of a computer mouse (Experiments 1, 2, and 5), a keyboard (Experiment 3), or a trackball (Experiment 4). We found that the flash-lag effect was significantly reduced when the observer was familiar with the directional relationship between the mouse movement and stimulus movement on a front parallel display (Experiments 1 and 2) and that, although the unfamiliar directional relationship between the mouse movement and stimulus movement increased the flash-lag effect at the beginning of the experimental session, the repetitive observation with the same unfamiliar directional relationship reduced the flash-lag effect (Experiment 5). We found no consistent reduction of the flash-lag effect with the use of a keyboard or a trackball (Experiments 3 and 4). These results suggest that the learning of a specific directional relationship between a proprioceptive signal of hand movements and a visual signal of stimulus movements is necessary for the reduction of the flash-lag effect.
\end{abstract}

When a flash is presented physically aligned with a continuously moving stimulus, the flash is perceived in a lagged position relative to the moving stimulus; this is called the flash-lag effect (Nijhawan, 1994). Such an illusory lag effect has been found not only for positional change, but also for changes in other visual attributes, such as changes in luminance, shape, and randomness (Sheth, Nijhawan, \& Shimojo, 2000). The flash-lag effect was initially explained as compensation for the intrinsic delay of visual processing (Nijhawan, 1994, 2002). Other researchers have explained the flash-lag effect as related to difference in latencies for the moving stimulus and the stationary flash (see, e.g., Bachmann, Luiga, Põder, \& Kalev, 2003; Krekelberg \& Lappe, 2001; Patel, Ogmen, Bedell, \& Sampath, 2000; Whitney \& Murakami, 1998; Whitney, Murakami, \& Cavanagh, 2000), or as the misperception of the location of the moving stimulus induced by the flash stimulus that resets motion integration (e.g., Eagleman \& Sejnowski, 2000a, 2000b). Regardless of the explanation of the effect, the phenomenon is thought to be a consequence of temporal and spatial aspects of visual processing for stimulus motion and other types of stimulus change.

The present study used the flash-lag effect to investigate the effects on visual perception of several factors related to observers' active control of visual stimuli. In most of the previous studies of the flash-lag effect, observers passively viewed the visual stimuli without any active involvement with stimulus change. However, Nijhawan and Kirschfeld (2003) demonstrated that there is a flash-lag effect related to the motor control system. In the present study, we measured the flash-lag effect in various conditions involving different types of observers' active manipulation of devices (computer mouse, keyboard, or trackball), controlling spatiotemporal aspects (i.e., movement) of the visual stimuli. By investigating the effects of the active control of stimulus motion on the flash-lag phenomenon, we aimed to increase knowledge about the function of active control in visual processing, rather than to understand the basis of the flash-lag effect.

Several recent studies have demonstrated that active observation of a visual stimulus can reduce the flash-lag effect. For instance, López-Moliner and Linares (2006) reported that the flash-lag effect was reduced when the observer's keypress controlled the presentation of the flash. They proposed that this reduction was caused by the effective utilization of attention because the observer could predict the presentation of the flash. Several previous studies have found that taking attention away from the flash (Baldo, Kihara, Namba, \& Klein, 2002; Murakami, 2001) or from the moving stimuli (Shioiri, Yamamoto, \& Yaguchi, 2002) enhances the flash-lag effect. The results of these previous

M. Ichikawa, ichikawa@L.chiba-u.ac.jp 
studies suggest that attention to both the moving stimulus and the flash may facilitate visual processing and reduce the flash-lag effect in active observation.

In our previous studies, we found that when the flash was presented with random timing and there was therefore no advantage of the prediction of the flash, the flash-lag effect was reduced if the observer actively controlled the continuous change of the visual stimulus by the use of the computer mouse (Ichikawa \& Masakura, 2004, 2006a, $2006 \mathrm{~b}$ ). Moreover, we obtained this reduction of the flashlag effect when the observers' mental set was that they were controlling the movement or the stimulus change, although the stimulus moved automatically. In one of our previous studies (Ichikawa \& Masakura, 2006a), we measured the reaction time (RT) for the shape change of the moving stimulus and flash, and we found that active observation reduced the RTs for both the moving stimulus and flash. Therefore, we proposed that the active control of stimulus change functions to facilitate and speed up processing for the whole visual field.

The results of our previous studies (Ichikawa \& Masakura, 2004, 2006a, 2006b) suggest that observers' manual operations to control the stimuli facilitate visual processing and thereby reduce the flash-lag effect. However, in those previous studies, we did not investigate whether the effect on visual processing depends on factors related to the specific type of manual operation. Thus, we were not sure whether we might generalize the reduction of the flash-lag effect to any kind of active manipulations of devices for stimulus change. Therefore, we conducted five experiments in which we varied the directional relationship between the manual movement and the stimulus movement, and the types of manual operation.

First, we investigated the directional relationship between stimulus movement and hand movement. In a previous study (Ichikawa \& Masakura, 2006a), we matched an upward stimulus movement on the display with a forward (away from the body) hand movement on the desk, and a downward stimulus movement with a backward (toward the body) hand movement. This directional matching is used in most computer operating systems (e.g., Microsoft's Windows, Apple's Mac OS, Linux). In the present study, we examined how inverting this directional relationship would affect the flash-lag effect in the first four experiments, and whether the learning of the unfamiliar directional relationship between the mouse and stimulus movements would reduce the flash-lag effect in the fifth experiment.

The second factor that we investigated was the type of hand movement. In previous studies, we used the computer mouse, which required the observers to continuously move an arm to control the stimulus movement on the display (Ichikawa \& Masakura, 2004, 2006a, 2006b). In the present experiments, we examined whether the reduction of the flash-lag effect required such a continuous arm movement. In the first, second, and fifth experiments, observers used the computer mouse to control stimuli, as in the previous studies. In the third experiment, observers controlled the stimulus by the use of a sustained keypress, which required the observer to avoid arm movement. In the fourth experiments, observers controlled the stimulus movement by the use of a trackball, which was manipulated by the fingers and palm.

In the present study, we examined how the directional relationship between the stimulus and hand movements and the types of hand movement would affect the flash-lag effect. One may predict that if the observer's having the mental set that he or she controls the stimulus movement is a sufficient condition for the reduction of the flash-lag effect, then we should obtain a reduction of the flash-lag effect regardless of these factors. However, our results indicated that this prediction was wrong. In accordance with the results of five experiments, we will discuss how the factors related to active movement affect visual processing.

\section{EXPERIMENT 1}

In Experiment 1, we investigated whether the directional relationship between the stimulus movement on the display and the observer's hand movement with a computer mouse would affect the flash-lag effect. We examined how reversing the directional relationship from the one that was used in our previous studies (Ichikawa \& Masakura, 2004, 2006a, 2006b), as well as in popular computer operating systems, would affect the reduction of the flash-lag effect. We applied the same method as the one used in our previous study (Ichikawa \& Masakura, 2006a), except that the present Experiment 1 included a new condition in which the directional relationship between the stimulus movement and the observer's hand movement was reversed. The flash-lag effect was defined as the temporal lag between the moving stimulus and the flash that was required for the perception that the stimulus and flash were visually aligned.

\section{Method}

Observers. One of the authors (M.I.) and 7 naive graduate students served as observers ( 2 females and 6 males). Their ages ranged from 21 to 39 years. All of them had normal or corrected-to-normal visual acuity and were right-handed. They had been using a personal computer with a computer mouse for at least 4 years.

Stimuli and Apparatus. A personal computer (Apple Macintosh G4) presented the stimuli on a 21-in. display (Eizo T962, $75 \mathrm{~Hz}$ ). The viewing distance was about $50 \mathrm{~cm}$. The observer sat on a chair in front of a desk ( $80 \mathrm{~cm}$ in height), with his or her head fixed on a chinrest, and grasped the computer mouse (Apple Pro Mouse M5769) with the right hand on the desk (Figure 1). A computer keyboard (Sanwa Supply SKB-M1090H) was placed by the observer's left hand.

The center of the display was at the eye level of the observer. As a fixation point, a red rectangle $(19.1 \times 19.0 \mathrm{arc} \min )$ was presented at the center of the display on a black background $\left(1.0 \mathrm{~cd} / \mathrm{m}^{2}\right)$. A white horizontal line $\left(334.3 \times 2.4 \mathrm{arc} \mathrm{min}, 87.6 \mathrm{~cd} / \mathrm{m}^{2}\right)$ was presented at the bottom or top of the display (about 15 arc deg above or below the fixation point) as the goal line for the moving stimulus. A red horizontal line $(334.3 \times 2.4$ arc $\mathrm{min})$ was presented at the bottom or top of the display (about 15.2 arc deg above or below the fixation point) to indicate the start position for the mouse. The moving stimulus and flash stimulus were white rectangles $\left(19.1 \times 19.0 \mathrm{arc} \mathrm{min}, 87.6 \mathrm{~cd} / \mathrm{m}^{2}\right)$. The moving stimulus went upward or downward along a linear course at 2.6 arc deg to the right or left of the vertical centerline of the display. The length of the movement trajectory for the moving stimulus was 28.8 arc deg. The vertical position lag between the moving stimulus and the flash ranged from -76.0 to 76.0 arc min in 19.0 arc min steps (negative or positive values indicate that the position of the flash was 


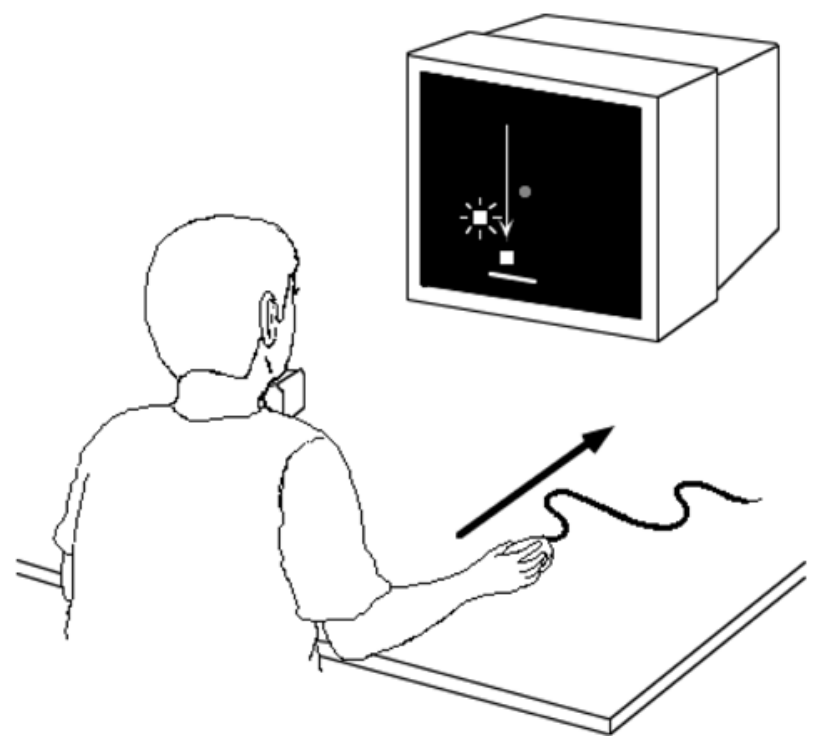

Figure 1. Apparatus for Experiment 1. In the inconsistent-motion condition, the forward (or backward) movement of the computer mouse on the desk was coupled with the downward (or upward) movement of the moving stimulus.

behind or ahead of the moving stimulus, respectively). That is, there were nine possible positions for the flash.

Procedure. There were three observation conditions in which the moving stimulus was controlled in different ways. In the first two conditions, the vertical position of the moving stimulus was controlled by the computer mouse that the observers manually moved forward (away from the body) or backward (toward the body) on the desk, whereas in the third condition, the stimulus moved automatically.

The first condition was the consistent-motion condition. The directional relationship between stimulus movement and hand movement was the same as the one used for the manual condition in our previous study (Ichikawa \& Masakura, 2006a), as well as for most computer operating systems. In this condition, the upward (and downward) movement of the moving stimulus was coupled with the forward (and backward) movement of the observer's hand. The second condition was the inconsistent-motion condition. In this condition, the directional relationship between stimulus movement and hand movement was opposite to the one used in the consistentmotion condition. In these two conditions, about $27.0 \mathrm{~cm}$ of mouse movement on the desk corresponded to 28.8 arc deg of vertical movement of the stimulus on the display. The observers could see their hands in the bottom of their visual field while viewing the fixation point. The observation for these two conditions was conducted in the same block. Observers were instructed to fixate the red point and to move the mouse (and thus the cursor on the display, which was the stimulus) for about $2 \mathrm{sec}$ from the start position to the goal line with a constant velocity while viewing the stimulus. If the movement took longer than $3,200 \mathrm{msec}$ or less than $1,600 \mathrm{msec}$, the experimenter told the observer that the movement was out of the acceptable range and that he or she should move the mouse faster or slower. That trial was presented again at the end of a block. Doing this meant that the mean velocity was restricted between 9.0 and $18.0 \mathrm{arc} \mathrm{deg} / \mathrm{sec}$ in each trial. In order to learn both the acceptable mouse movement rate and that the mouse moved the stimulus, observers had a training session with at least 40 trials before the experimental trials until the observer's mouse movement was within the acceptable range (from 1,600 to $3,200 \mathrm{msec}$ ) in at least 10 consecutive trials. In the training session, observers moved the mouse while viewing the display that showed the moving stimulus with the fixation point and goal line, but no flash stimulus.
The third condition was the automatic condition, in which the observer viewed the stimulus without using the mouse. The sessions for this condition were conducted after the sessions for the consistent-motion and inconsistent-motion conditions. Before the experimental sessions, the observers had at least 10 training trials until they felt that they understood the task. The stimulus moved with a constant velocity that was determined by the mean velocity from the consistent-motion and inconsistent-motion conditions for each individual. After a random interval that ranged from 1,000 to $2,000 \mathrm{msec}$ after the observer pressed the space key, the stimulus started to move with a constant velocity.

There were five blocks for the consistent-motion and inconsistentmotion conditions. In each block, 72 stimulus conditions [directional relationship between the hand and stimulus motions $(2) \times$ lag between the stimuli (9) $\times$ direction of the stimulus movement $(2) \times$ horizontal position of the moving stimulus (2)] were presented in random order. There were also five blocks for the automatic condition. In each block, 36 stimulus conditions [lag between the stimuli (9) $\times$ direction of the stimulus movement $(2) \times$ horizontal position of the moving stimulus (2)] were presented in random order. At the beginning of each trial, the fixation point, the red line, and the white goal line were presented. In accordance with the position of the red line, the observers located the computer mouse at the start point on the desk for the consistent-motion and inconsistent-motion conditions. When the observers pressed the space key to start the trial, the stimulus was presented at the bottom or top of the display (the start point for the trial). In each condition, the observer's mouse control (consistent-motion or inconsistent-motion condition) or keypress (automatic condition) started the vertical movement of the stimulus. During the stimulus movement between the levels of the fixation point and goal line, the flash was presented for $13 \mathrm{msec}$ at one of the nine possible positions. After the moving stimulus reached the goal line, the observers pressed a key to report whether the flash was above or below the moving stimulus.

After all of the experimental sessions, the observers reported the easiest and most difficult conditions and guessed the conditions in which their judgment was the most and least accurate. They also reported how they had felt during the sessions for each condition.

\section{Results and Discussion}

For all of the sessions, the mean amount of time that each observer took in moving the mouse from the start point to the goal ranged from 2,328 to $2,561 \mathrm{msec}$ (for all observers, $M=2,420 \mathrm{msec}$ ) for the consistent-motion condition, and from 2,217 to $2,468 \mathrm{msec}(M=2,354 \mathrm{msec})$ for the inconsistent-motion condition. Because the flash-lag effect depends on the velocity of the moving stimulus (Brenner \& Smeets, 2000; Krekelberg \& Lappe, 2000; Nijhawan, 1994), we examined whether the velocity of the moving stimulus would affect the flash-lag effect in the consistentmotion and inconsistent-motion conditions. The mean of the velocities for each observer ranged from 11.2 to 12.3 $\operatorname{arc~deg} / \mathrm{sec}(M=11.8 \mathrm{arc} \mathrm{deg} / \mathrm{sec})$ for the consistentmotion condition, and from 11.6 to $13.0 \mathrm{arc} \mathrm{deg} / \mathrm{sec}(M=$ $12.2 \mathrm{arc} \mathrm{deg} / \mathrm{sec}$ ) for the inconsistent-motion condition. There was no consistent difference in the velocity between the consistent-motion and inconsistent-motion conditions. The standard deviation of the velocity within an individual observer ranged from 0.92 to $1.31 \mathrm{arc} \operatorname{deg} / \mathrm{sec}(M=1.13$ $\operatorname{arc~deg} / \mathrm{sec}$ ) for the consistent-motion condition, and from 0.88 to $1.45 \mathrm{arc} \mathrm{deg} / \mathrm{sec}(M=1.13 \mathrm{arc} \mathrm{deg} / \mathrm{sec})$ for the inconsistent-motion condition. These small standard deviations indicate that the observer obeyed the instruction to move the mouse with a constant and stable velocity in the trials for these conditions. The velocity for the automatic 
condition was determined by the mean velocity from the consistent-motion and inconsistent-motion conditions for each individual; it ranged from 11.4 to $12.5 \mathrm{arc} \mathrm{deg} / \mathrm{sec}$ $(M=12.0 \mathrm{arc} \mathrm{deg} / \mathrm{sec})$.

Figure 2A shows the results for 1 naive observer, as an example. The vertical axis indicates the frequency of the observer's report that the moving stimulus passed the level of the flash. The horizontal axis shows the physical lag between the moving stimulus and the flash. Zero on the horizontal line indicates that the moving stimulus and flash were presented at the same vertical level. Therefore, the veridical judgment at this point was $50 \%$. In this condition, this observer judged that the moving stimulus passed the fixation point in about $75 \%$ of the trials in the inconsistentmotion and automatic conditions, whereas the judgment was close to $50 \%$ in the consistent-motion condition.

As in the previous studies (Ichikawa \& Masakura, 2004, 2006a, 2006b), the flash-lag effect was derived from the duration that each observer took in moving the stimulus from the start to the goal, with a position lag that the probit analysis (Finney, 1971) determined to be the 50\% threshold for the response that the moving stimulus had passed the level of the flash. (We found almost the same results in the following analyses, even if the flash-lag effect was derived from the spatial lag between the moving stimulus and the flash in all of the experiments.) In calculating the flash-lag effect for each observer, we used the mean velocity from all of the sessions for each of the consistent-motion and inconsistent-motion conditions. For the consistent-motion condition, the means of the flash-lag effect for the stimuli presented in the left and right visual fields were, respectively, 38.7 and $26.9 \mathrm{msec}$, whereas those for the stimuli presented in the left and right visual fields for the inconsistent-motion condition were, respectively, 66.8 and $45.5 \mathrm{msec}$. For the automatic condition, the means of the flash-lag effect for the stimuli presented in the left and right visual fields were, respectively, 56.6 and $48.8 \mathrm{msec}$. For the consistent-motion condition, the means of the flash-lag effect for the upward and downward stimulus movements were, respectively,
26.1 and $38.3 \mathrm{msec}$, whereas for the inconsistent-motion condition, those for the upward and downward stimulus movements were, respectively, 42.3 and $66.2 \mathrm{msec}$. For the automatic condition, the means of the flash-lag effect for the upward and downward stimulus movements were, respectively, 45.0 and $59.7 \mathrm{msec}$. We found no consistent difference in the flash-lag effect between the left and right visual fields for the consistent-motion condition $[t(7)=$ $1.117, p>.10]$, the inconsistent-motion condition $[t(7)=$ $1.284, p>.10]$, and the automatic condition $[t(7)=0.901$, $p>.10]$, or between the upward and downward stimulus movement for the consistent-motion condition $[t(7)=$ $0.593, p>.10]$, the inconsistent-motion condition $[t(7)=$ $1.048, p>.10]$, and the automatic condition $[t(7)=1.152$, $p>.10]$. The data from these conditions were combined in the following analyses.

Figure $2 \mathrm{~B}$ shows the means of the $50 \%$ thresholds in each condition for the 8 observers. Figure $2 \mathrm{~B}$ shows that the flash-lag effect for the consistent-motion condition was about three fifths of that for the automatic condition, whereas that for the inconsistent-motion condition was at the same level as that for the automatic condition.

We conducted a one-way repeated measures ANOVA for the three conditions using the data from the 8 observers (Figure 2B). The main effect of condition was significant $[F(2,14)=9.198, p<.005]$. Tukey's post hoc HSD tests show that the flash-lag effect in the consistentmotion condition was significantly smaller than it was in the other two conditions $(p<.05)$.

These results indicate that observers' manual control of the stimulus movement in the consistent-motion condition reduced the flash-lag effect, whereas the manual control in the inconsistent-motion condition did not have such an effect. These findings suggest that the reduction of the flashlag effect in the consistent-motion condition depended not only on the observers' activity in controlling the stimulus movement, but also on the directional relationship between the stimulus movement on the display and the active manipulation of the mouse movement on the desk.
A

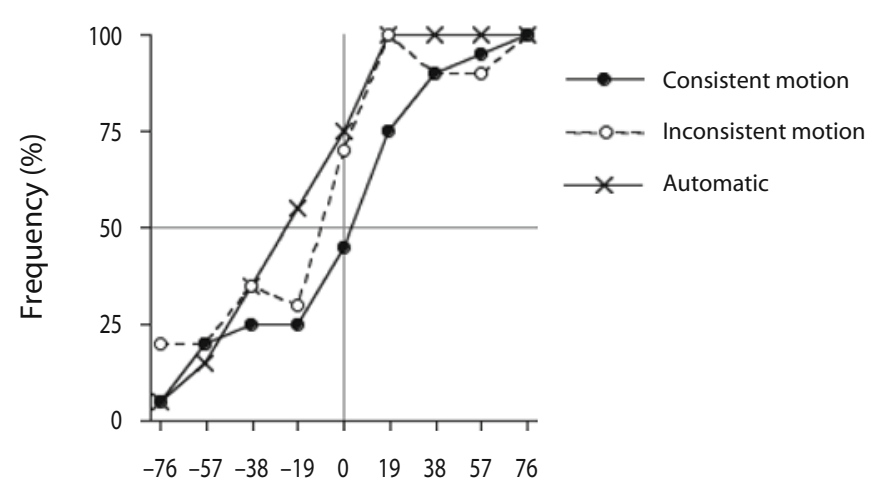

Position Lag (arc min)
B

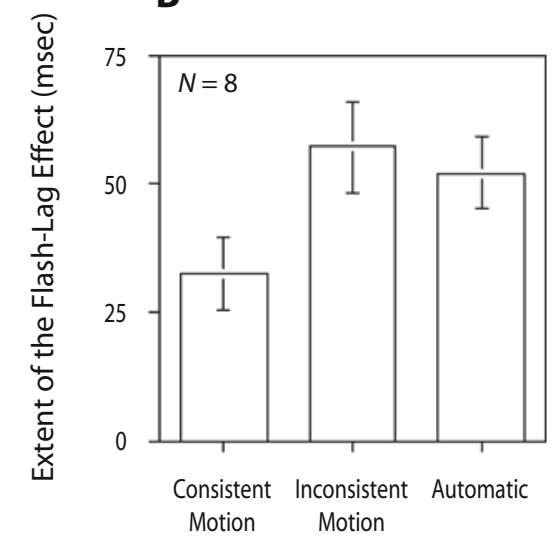

Condition

Figure 2. Results of Experiment 1. (A) Example of an observer. (B) Means and $S E$ s of the $50 \%$ thresholds for the three conditions. 
There were inconsistencies between the results of the analysis of the flash-lag effect and observers' introspective reports. As in our previous study (Ichikawa \& Masakura, 2006a), all of the naive observers reported that the automatic condition was the easiest condition, and they predicted that their performance was the most accurate in the automatic condition. As was also found in our previous study, however, the most accurate condition was the consistent-motion condition. In addition, all of the observers reported that they felt that they had controlled the stimulus movement in both the consistent-motion and inconsistent-motion conditions. There were no subjective differences between the consistent-motion and inconsistent-motion conditions in difficulty in controlling the stimulus movement and in judging the relative position of the flash and the moving stimulus. Surprisingly, however, there was a significant difference in the flash-lag effect between these two conditions. This result suggests that the observers' subjective mental set that they controlled the stimulus movement was not a sufficient condition for the reduction of the flash-lag effect; instead, the directional relationship between the stimulus movement and hand movement was critical.

\section{EXPERIMENT 2}

In Experiment 1, we found that the observer's manual control reduced the flash-lag effect when the upward (or downward) movement of the moving stimulus was coupled with the forward (or backward) movement of the observer's hand. In Experiment 1, the observer could see his or her hand in controlling the stimulus movement in the bottom of their visual field. Therefore, the image of the observer's hand moved consistently or inconsistently with the stimulus movement on the display. In Experiment 2, we examined whether the visibility of the observer's hand that controlled the mouse and that moved consistently with stimulus movement on the display would be necessary for the reduction of the flash-lag effect in the consistent-motion condition.

\section{Method}

Observers. One of the authors (M.I.) and 3 new, naive graduate students served as observers. Their ages ranged from 22 to 41 years. All of them had normal or corrected-to-normal visual acuity.

Stimuli and Apparatus. A black styrene board $(90 \times 180 \times$ $0.5 \mathrm{~cm}$ ) stood on the desk at the right side of the observer. The observers could not see their right hands, which controlled the mouse during the trial, because the board covered them. The other settings and apparatus were the same as those in Experiment 1.

Procedure. The procedures were the same as those used in Experiment 1 . There were three observation conditions, as in the previous experiment: the consistent-motion condition, the inconsistentmotion condition, and the automatic condition.

\section{Results and Discussion}

For all of the sessions, the mean amount of time that each observer took in moving the mouse from the start point to the goal ranged from 2,383 to $2,490 \mathrm{msec}(M=2,440 \mathrm{msec})$ for the consistent-motion condition, and from 2,342 to $2,452 \mathrm{msec}(M=2,416 \mathrm{msec})$ for the inconsistent-motion condition. The mean velocity for each observer ranged from 11.5 to $12.1 \mathrm{arc} \mathrm{deg} / \mathrm{sec}(M=11.8 \mathrm{arc} \mathrm{deg} / \mathrm{sec})$ for the consistent-motion condition, and from 11.7 to 12.3 arc deg $/ \mathrm{sec}(M=11.9 \operatorname{arc~deg} / \mathrm{sec})$ for the inconsistentmotion condition. These values were at the same level as those obtained in Experiment 1. There was no consistent difference in the velocity between the consistent-motion and the inconsistent-motion conditions. The standard deviation of the velocity for each observer ranged from 0.77 to $1.15 \mathrm{arc} \mathrm{deg} / \mathrm{sec}(M=0.96 \mathrm{arc} \mathrm{deg} / \mathrm{sec})$ for the consistentmotion condition, and from 0.76 to $1.10 \mathrm{arc} \mathrm{deg} / \mathrm{sec}(M=$ $0.97 \mathrm{arc} \mathrm{deg} / \mathrm{sec}$ ) for the inconsistent-motion condition. The velocity for the automatic condition, which was determined by the mean velocity from the consistent-motion and inconsistent-motion conditions for each individual, ranged from 11.7 to $12.2 \mathrm{arc} \mathrm{deg} / \mathrm{sec}(M=11.9 \mathrm{arc} \mathrm{deg} / \mathrm{sec})$.

For the consistent-motion condition, the means of the flash-lag effect for the stimuli presented in the left and right visual fields were, respectively, 17.2 and $13.3 \mathrm{msec}$, whereas for the inconsistent-motion condition, those for the stimuli presented in the left and right visual fields were, respectively, 30.3 and $23.4 \mathrm{msec}$. For the automatic condition, the means of the flash-lag effect for the stimuli presented in the left and right visual fields were, respectively, 36.8 and $30.9 \mathrm{msec}$. For the consistent-motion condition, the means of the flash-lag effect for the upward and downward stimulus movements were, respectively, 7.9 and $19.9 \mathrm{msec}$, whereas in the inconsistent-motion condition, those for the upward and downward stimulus movements were, respectively, 31.6 and $27.0 \mathrm{msec}$. For the automatic condition, the means of the flash-lag effect for the upward and downward stimulus movements were, respectively, 36.4 and $31.3 \mathrm{msec}$. We found no consistent difference in the flash-lag effect between the left and right visual fields for the consistent-motion condition $[t(3)=0.601, p>$ $.10]$, the inconsistent-motion condition $[t(3)=0.902, p>$ $.10]$, and the automatic condition $[t(3)=2.213, p>.10]$, or between the upward and downward stimulus movement for the consistent-motion condition $[t(3)=0.400, p>$ $.10]$, the inconsistent-motion condition $[t(3)=0.138, p>$ $.10]$, and the automatic condition $[t(3)=0.348, p>.10]$. The data from these conditions were combined in the following analyses.

Figure 3 shows the means of the $50 \%$ thresholds in each condition for the 4 observers. The values for the three conditions look smaller than those obtained in Experiment 1, although the velocities of the stimulus movement in these experiments were at similar levels. Because the flash-lag effect was smaller than that in Experiment 1, even in the automatic condition, we assume that the difference in the level of the flash-lag effect between these experiments was caused by individual variance of the observers who took part in each experiment.

Introspective reports from the observers were very similar to those in Experiment 1. All of the naive observers reported that the automatic condition was the easiest condition, and they predicted that their performance was the most accurate in the automatic condition. All of them reported that there were no subjective differences between the consistent-motion and inconsistent-motion conditions 


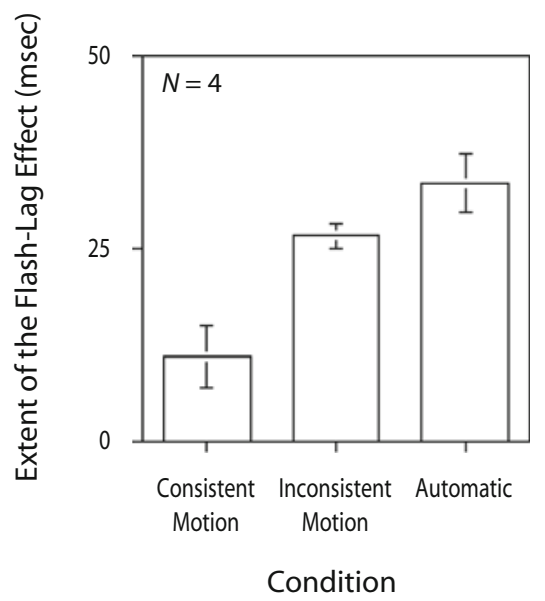

Figure 3. Results of Experiment 2. Means and SEs of the 50\% threshold for the three conditions.

in difficulty in controlling the stimulus movement and in judging the relative position of the flash and the moving stimulus. Figure 3 shows, however, that the flash-lag effect for the consistent-motion condition was about one third of that for the automatic condition, whereas that for the inconsistent-motion condition was at a level similar to that for the automatic condition. An ANOVA for the data from the 4 observers found a significant main effect $[F(2,6)=$ $10.073, p<.05]$. Tukey's post hoc HSD tests showed that the flash-lag effect in the consistent-motion condition was significantly smaller than those in the inconsistent-motion and automatic conditions $(p<.05)$.

These results indicate that the visibility of the observer's hand, which was moving consistently or inconsistently with the stimulus movement on the display, had no significant effect on the flash-lag effect. We propose that the proprioceptive signal of the hand movement, rather than the visual signal of the hand movement, was important for the reduction of the flash-lag effect in the consistentmotion condition.

\section{EXPERIMENT 3}

In Experiments 1 and 2, we found that the observer's manual control reduced the flash-lag effect when the upward (or downward) movement of the moving stimulus was coupled with the forward (or backward) movement of the observer's hand. In Experiment 3, we examined whether the observer's continuous manual movement would be necessary for the reduction of the flash-lag effect, or whether the observer's keypress would reduce the flash-lag effect. In Experiment 3, we compared the flashlag effect in the condition in which observers controlled the stimulus movement by a keypress with that in a condition in which the stimulus moved automatically.

\section{Method}

Observers. One of the authors (M.I.) and 7 naive graduate students served as observers. Five of them had participated in the first experiment. Their ages ranged from 21 to 39 years. All of them had normal or corrected-to-normal visual acuity.
Stimuli and Apparatus. We used the same setup of the equipment and the stimulus configuration as in Experiment 1.

Procedure. There were three observation conditions, as in Experiment 1 . In the consistent-motion condition, the upward movement of the stimulus was coupled with continuously pressing the "J" key on the keyboard with the right middle finger, and the downward movement of the stimulus was coupled with continuous pressing of the "N" key with the right index finger. Because the keyboard was fixed in front of the observer, the middle finger was farther than the index finger from the observer's body. In the inconsistent-motion condition, the directional relationship between the keypressing and stimulus movement was opposite of that in the consistent-motion condition. In these two conditions, the stimulus moved during the keypressing with a constant velocity of 12.0 arc $\mathrm{deg} / \mathrm{sec}$, which was the mean velocity from all of the sessions for the consistent-motion and inconsistent-motion conditions in Experiment 1. The observers could see their hands and keyboard in the bottom of their visual field while viewing the fixation point. The sessions for the consistent-motion and inconsistent-motion conditions were conducted in the same block as in Experiment 1. At the beginning of each trial, the fixation point, the red line, and the white line were presented. In accordance with the position of the red line, the observer's pressing of the "J" or "N" key started the vertical movement of the stimulus, and the stimulus continued to move during the continuous keypress. In order to become familiar with the task and to learn that the continuous keypressing moved the stimulus, observers had a training session with at least 40 trials before the experimental trials. In the training session, observers pressed and released the key sometimes to make sure that their keypressing moved the stimulus while viewing the display that showed the moving stimulus with the fixation point and goal line but no flash stimulus. In the experimental sessions, they were instructed not to release the key after the stimulus passed the vertical level of the fixation point, to avoid the disruption of the smooth stimulus movement.

The other procedures were the same as those used in Experiment 1 .

\section{Results and Discussion}

For the consistent-motion condition, the means of the flash-lag effect for the stimuli presented in the left and right visual fields were, respectively, 44.9 and $45.4 \mathrm{msec}$, whereas those for the stimuli presented in the left and right visual fields for the inconsistent-motion condition were, respectively 42.3 and $25.9 \mathrm{msec}$. Those for the stimuli presented in the left and right visual fields for the automatic condition were, respectively, 48.6 and $40.8 \mathrm{msec}$. For the consistent-motion condition, the means of the flash-lag effect for the upward and downward stimulus movements were, respectively, 42.4 and $63.1 \mathrm{msec}$, whereas those for the upward and downward stimulus movements for the inconsistent-motion condition were, respectively, 24.9 and $40.8 \mathrm{msec}$. Those for the upward and downward stimulus movements for the automatic condition were, respectively, 38.2 and $50.2 \mathrm{msec}$. We found no consistent difference in the flash-lag effect between the left and right visual fields for the consistent-motion condition $[t(7)=0.041, p>$ $.10]$, the inconsistent-motion condition $[t(7)=1.233, p>$ $.10]$, and the automatic condition $[t(7)=0.960, p>.10]$, or between the upward and downward stimulus movement for the consistent-motion condition $[t(7)=0.638, p>$ $.10]$, the inconsistent-motion condition $[t(7)=0.968, p>$ $.10]$, and the automatic condition $[t(7)=1.083, p>.10]$. The data from these conditions were combined in the following analyses. 


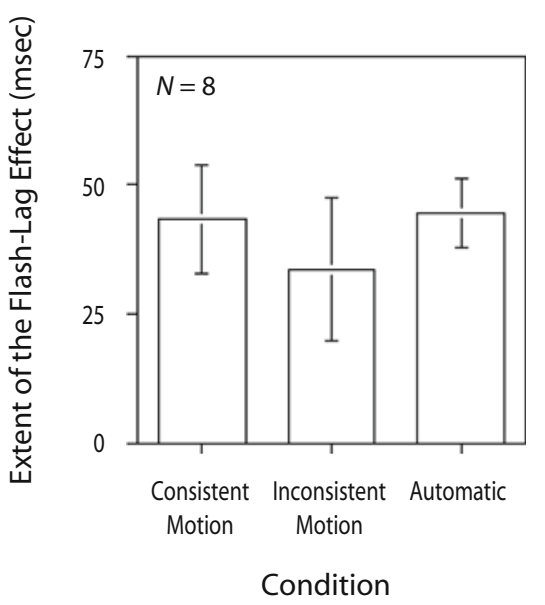

Figure 4. Results of Experiment 3. Means and SEs of the 50\% threshold for the three conditions.

The flash-lag effect was derived from the same duration $(2,386 \mathrm{msec})$ that observers took in moving the stimulus with a constant velocity of $12.0 \mathrm{arc} \mathrm{deg} / \mathrm{sec}$ and the position lag for the perceptual alignment between the moving stimulus and flash. Figure 4 shows the means of the flashlag effect in each condition for the 8 observers. Figure 4 shows that there was no consistent reduction of the flashlag effect for the active observation. A one-way repeated measures ANOVA for the three conditions using the data from the 8 observers showed that the main effect of the condition was not significant $[F(2,14)=1.600, p>.10]$.

In the consistent-motion and inconsistent-motion conditions, all of the observers reported that they felt that they were controlling the stimulus movement by continuously pressing the key. In the automatic condition, they felt that they had no effect on the stimulus movement and that the stimulus moved automatically, independently of their keypressing. There was no consistent difference in the observers' introspective reports about the difficulty of the task in the three conditions. Four naive observers who had taken part in Experiment 1 reported that the trials in Experiment 3 were much easier than those in Experiment 1, because they could concentrate their judgments on the relationship between the moving stimulus and the flash without paying attention to the constant hand movement within the restricted interval. The results of the analysis indicate that the observer's voluntary keypressing cannot reduce the flash-lag effect.

\section{EXPERIMENT 4}

The results of the previous three experiments indicate that the reduction of the flash-lag effect depends on the method of active control of the visual stimulus. In Experiment 3 , there was no significant reduction of the flash-lag effect, in contrast with Experiments 1 and 2, in which coupling the forward (or backward) mouse movement with the upward (or downward) movement of the stimulus reduced the flash-lag effect. These results suggest that continuous manual movement in which the direction is coupled with a specific direction of stimulus movement is required for the reduction of the flash-lag effect. In Experiment 4, we examined whether the reduction of the flash-lag effect in terms of the observers' active control might be generalized to continuous control of a trackball manipulated by the fingers and palm. In Experiment 4, we compared the flash-lag effect that was obtained in the condition in which observers controlled the stimulus movement by the use of a trackball with that in the condition in which the stimulus moved automatically.

\section{Method}

Observers. Eight observers who had taken part in Experiment 3 participated in Experiment 4. Only 1 observer (M.I.) was familiar with the trackball.

Stimuli and Apparatus. The setup of the equipment and the stimulus configuration were the same as those in Experiment 1, except that the observers manipulated a trackball (Logitech, Cordless Optical TrackMan CT-100) with the fingers and palm of the right hand to control the stimulus movement. The trackball was fixed on the desk in front of the observers (Figure 5).

Procedure. As in Experiment 1, there were three observation conditions: the consistent-motion condition, the inconsistent-motion condition, and the automatic condition. In the consistent-motion condition, the upward (or downward) movement of the moving stimulus was coupled with the forward (or backward) movement of the observer's fingers and palm (Figure 5). In the inconsistent-motion condition, the directional relationship between the hand movement and stimulus movement was the opposite of the one used in the consistent-motion condition. In these two conditions, observers could move the stimulus with a one-stroke movement of palm and fingers; about $15 \mathrm{~cm}$ of fingers and the palm movement corresponded to $28.8 \mathrm{arc}$ deg of vertical movement of the stimulus on the display. They did not need to repeat the action to roll the ball in each trial to reach the goal line. This indicates that the movement of the finger and palm to control the trackball was approximately half of the hand movement used to control the mouse in Experiments 1 and 2. The other procedures were the same as those used in Experiment 1.

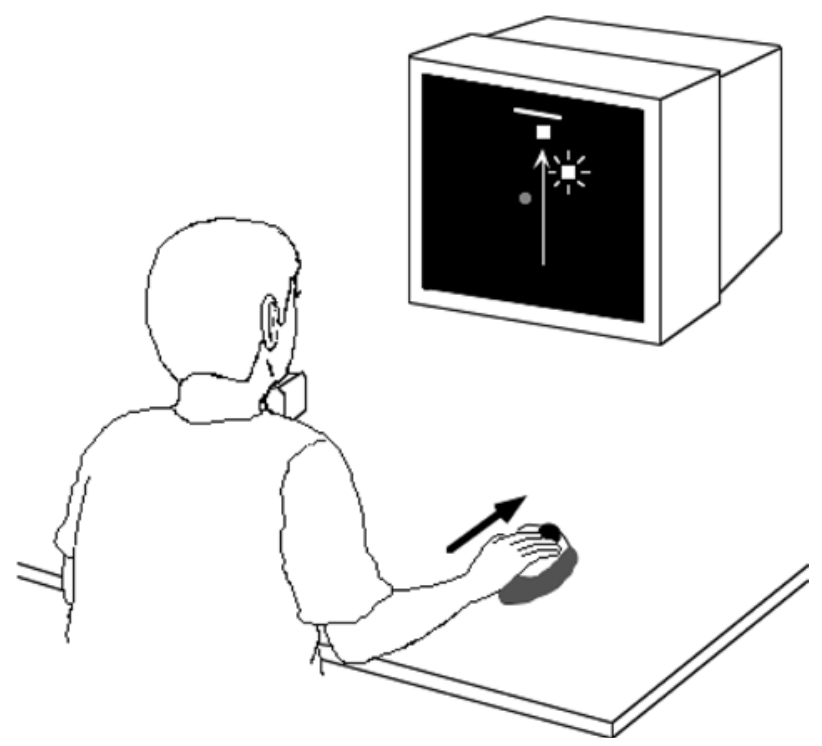

Figure 5. Apparatus for Experiment 4. In the consistent-motion condition, the forward (or backward) movement of the trackball on the desk was coupled with the upward (or downward) movement of the moving stimulus. 
In the automatic condition, the observer viewed the stimulus and flash without using the trackball. The procedures were the same as those for the automatic condition in the first experiment.

\section{Results and Discussion}

For all of the sessions, the mean of the times that each observer took in moving the hand from the start point to the goal ranged from 2,661 to $3,031 \mathrm{msec}(M=2,853 \mathrm{msec})$ for the consistent-motion condition, and from 2,498 to $2,976 \mathrm{msec}(M=2,779 \mathrm{msec})$ for the inconsistentmotion condition. The means of the velocities for each observer ranged from 9.5 to $10.8 \mathrm{arc} \mathrm{deg} / \mathrm{sec}(M=10.1$ $\operatorname{arc~deg} / \mathrm{sec}$ ) for the consistent-motion condition, and from 9.7 to $11.5 \mathrm{arc} \mathrm{deg} / \mathrm{sec}(M=10.4 \mathrm{arc} \mathrm{deg} / \mathrm{sec})$ for the inconsistent-motion condition. There was no consistent difference in the velocity between the consistent-motion and inconsistent-motion conditions. The standard deviation of the velocity within an individual observer ranged from 1.92 to $2.36 \mathrm{arc} \mathrm{deg} / \mathrm{sec}(M=2.15 \mathrm{arc} \mathrm{deg} / \mathrm{sec})$ for the consistent-motion condition, and from 1.82 to 2.44 arc deg/sec $(M=2.14 \mathrm{arc} \mathrm{deg} / \mathrm{sec})$ for the inconsistentmotion condition. Although these standard deviations were larger than those obtained in Experiments 1 and 2, they still remained at a low level.

For the consistent-motion condition, the means of the flash-lag effect in the left and right visual fields were, respectively, 48.3 and $32.3 \mathrm{msec}$, whereas those in the left and right visual fields for the inconsistent-motion condition were, respectively, 50.8 and $33.7 \mathrm{msec}$. For the automatic condition, the means of the flash-lag effect for the left and right visual fields were, respectively, 46.5 and $31.8 \mathrm{msec}$. For the consistent-motion condition, the means of the flashlag effect for the upward and downward stimulus movements were, respectively, 34.9 and $46.9 \mathrm{msec}$, whereas those for the upward and downward stimulus movements for the inconsistent-motion condition were, respectively, 30.5 and $51.6 \mathrm{msec}$. For the automatic condition, the means of the flash-lag effect for the upward and downward stimulus movements were, respectively, 32.0 and $47.5 \mathrm{msec}$. We found no consistent difference in the flash-lag effect between the left and right visual fields for the consistent-motion condition $[t(7)=1.671, p>.10]$, the inconsistent-motion condition $[t(7)=2.105, p>.05]$, and the automatic condition $[t(7)=1.423, p>.10]$, or between the upward and downward stimulus movement for the consistent-motion condition $[t(7)=0.435, p>.10]$, the inconsistent-motion condition $[t(7)=0.971, p>.10]$, and the automatic condition $[t(7)=0.959, p>.10]$. The data from these conditions were combined in the following analyses.

The flash-lag effect was derived in the same way as in the previous three experiments. Figure 6 shows the means of the $50 \%$ thresholds from the 8 observers in each condition. Figure 6 shows that there was no consistent reduction of the flash-lag effect related to active observation. A oneway repeated measures ANOVA for the three conditions using the data from the 8 observers showed that the main effect of the condition was not significant $[F(2,14)=$ $1.798, p>.10]$.

These results of the analysis imply that the observers' continuous hand movements controlling the stimu-

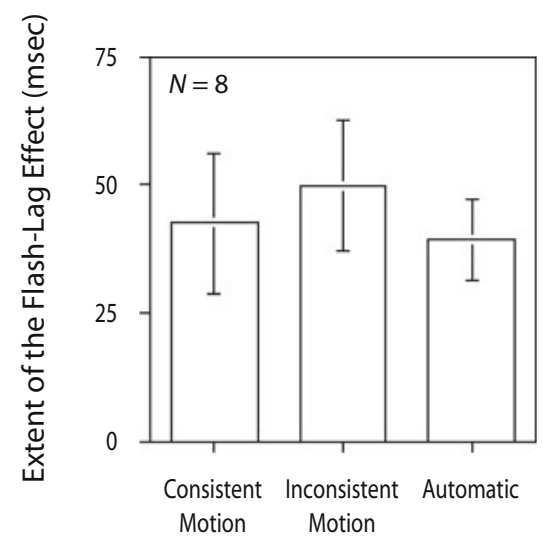

Condition

Figure 6. Results of Experiment 4. Means and $S E$ s of the $50 \%$ threshold for the three conditions.

lus movement by the use of a trackball did not reduce the flash-lag effect. These results indicate that the reduction of the flash-lag effect related to observers' active control could not be generalized to the continuous control using the trackball, and that the mouse control had an advantage in reducing the flash-lag effect, as compared with manipulating the other devices.

Although almost all of the observers were not familiar with the trackball as a device for manipulating the computer, all of the observers felt that they had controlled the stimulus movement by the use of the trackball in the consistent-motion and inconsistent-motion conditions. They reported, however, that moving the stimulus with a constant velocity required more effort using the trackball than did moving the stimulus by the use of the computer mouse or keypress.

Introspective reports from the observers were very similar to those in Experiment 1. That is, all of the observers reported that the automatic condition was the easiest condition, and they predicted that their performance was the most accurate in the automatic condition, although there was no significant difference in the flash-lag effect among these conditions.

\section{EXPERIMENT 5}

In Experiments 1 and 2, we found that the observer's manual control reduced the flash-lag effect only in the consistent-motion condition, in which the upward (or downward) movement of the moving stimulus was coupled with the forward (or backward) movement of the observer's hand, as in a popular computer operating system. This result suggests that the experience of a certain relationship between stimulus movement and hand movement is important for the reduction of the flash-lag effect in active observation. In Experiment 5, we examined whether the repetitive observation with the unfamiliar directional relationship between the stimulus movement and hand movement would affect the flash-lag effect. In the experiment, observers had 360 training trials in which the 
upward (or downward) movement of the moving stimulus was coupled with the backward (or forward) movement of the hand, as in the inconsistent-motion condition in Experiment 1.

\section{Method}

Observers. Five new naive graduate and undergraduate students served as observers. Their ages ranged from 20 to 24 years. All of them had normal or corrected-to-normal visual acuity.

Stimuli and Apparatus. The apparatus, the setup of the equipment, and the stimulus configuration were the same as those used in Experiment 1. The observers could see their hands in the bottom of their visual field while viewing the fixation point.

Procedure. There were two conditions. The first was the manual condition. The directional relationship between hand movement and stimulus movement was the same as the one that had been used for the inconsistent-motion condition in Experiment 1. The acceptable range for the hand movement was from 2,000 to 2,800 $\mathrm{msec}$, and it was narrower than that used in Experiment 1. The second condition was the automatic condition. The moving stimulus moved automatically. In each block for the manual and automatic conditions, 36 stimulus conditions [lag between the stimuli (9) $\times$ direction of the movement $(2) \times$ horizontal position of the moving stimulus (2)] were presented in random order. Other procedures for the manual and automatic conditions were the same as those used for the inconsistent-motion and automatic conditions in Experiment 1. In each trial for these conditions, the observers pressed a key to report whether the flash was above or below the moving stimulus.

After the observers had 1 block to learn the acceptable mousemovement rate in the manual condition, they had 5 blocks for the manual condition. Then they had 5 blocks for the automatic condition, which were followed by 10 blocks for the training sessions. In the training sessions, the observers were instructed to move the mouse in about $2,400 \mathrm{msec}$ from the start line to the goal line. If the movement took longer than $2,800 \mathrm{msec}$ or less than $2,000 \mathrm{msec}$, the experimenter told the observer that the movement was out of the acceptable range. In addition, if the time for the movement was within the range between 2,373 and 2,427 msec, the experimenter told the observer that the movement was in the center of the acceptable range. After 10 blocks for the training sessions ( 360 trials), the observers had another 5 blocks for the manual and automatic conditions.

\section{Results and Discussion}

For all of the sessions in the manual condition, the mean amount of time that each observer took in moving the mouse from the start point to the goal ranged from 2,394 to $2,491 \mathrm{msec}(M=2,435 \mathrm{msec})$ before the training sessions, and from 2,354 to $2,437 \mathrm{msec}(M=2,407 \mathrm{msec})$ after the training sessions. The mean of the velocities for each observer ranged from 11.5 to $12.0 \mathrm{arc} \mathrm{deg} / \mathrm{sec}(M=$ $11.8 \mathrm{arc} \mathrm{deg} / \mathrm{sec}$ ) before the training sessions, and from 11.8 to $12.2 \mathrm{arc} \mathrm{deg} / \mathrm{sec}(M=11.9 \mathrm{arc} \mathrm{deg} / \mathrm{sec})$ after the training sessions. These values were at the same level as those obtained in Experiment 1. The standard deviation of the velocity within an individual observer ranged from 0.55 to $0.81 \mathrm{arc} \mathrm{deg} / \mathrm{sec}(M=0.69 \mathrm{arc} \mathrm{deg} / \mathrm{sec})$ before the training sessions, and from 0.62 to $0.80 \mathrm{arc} \mathrm{deg} / \mathrm{sec}$ ( $M=0.71 \mathrm{arc} \mathrm{deg} / \mathrm{sec})$ after the training sessions. The mean frequency of the trials in which the time to move the mouse from the start point to the goal line was not within the acceptable range was $4.2 \%$ (for all of the observers, the standard deviation was $2.57 \%$ ) before the training sessions, and 2.2\% (standard deviation was $1.61 \%$ ) after the training sessions, and the difference was not significant.
These results indicate that the training had no explicit effects on the velocity of the mouse movement in the manual condition.

For the manual condition, the means of the flash-lag effect in the left and right visual fields were, respectively, 53.0 and $44.4 \mathrm{msec}$, whereas those for the left and right visual fields for the automatic condition were, respectively, 39.0 and $40.3 \mathrm{msec}$. For the manual condition, the means of the flash-lag effect for the upward and downward stimulus movements were, respectively, 54.9 and $51.0 \mathrm{msec}$, whereas those for the upward and downward stimulus movements for the automatic condition were, respectively, 34.0 and $44.5 \mathrm{msec}$. We found no consistent difference in the flash-lag effect between the left and right visual fields for the manual condition $[t(4)=1.544, p>$ $.10]$ and the automatic condition $[t(4)=0.272, p>.10]$, or between the upward and downward stimulus movement for the manual condition $[t(4)=0.175, p>.10]$ and the automatic condition $[t(4)=0.724, p>.10]$. The data from these conditions were combined in the following analyses.

Figure 7 shows the means of the $50 \%$ thresholds in each condition for the 5 observers. The values for the manual condition were larger than those for the automatic condition both before and after the training sessions. This result indicates that 360 trials in the training sessions with the unfamiliar directional relationship between hand movement and stimulus movement were not enough to reduce the flash-lag effect to a level that was smaller than that obtained in the automatic condition. Paired $t$ tests, however, showed that, for the manual condition, the flash-lag effect after the training sessions was significantly smaller than that before the training sessions $[t(4)=3.489, p<$ $.05]$, whereas, for the automatic condition, there was no significant difference between before and after the training sessions $[t(4)=0.992, p>.10]$. These results indicate that repetitive observation with the unfamiliar relationship between stimulus movement and hand movement

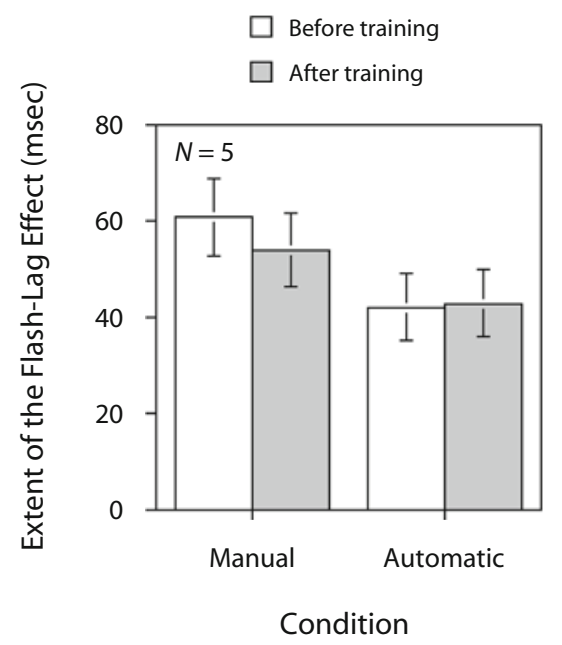

Figure 7. Results of Experiment 5. Means and SEs of the 50\% threshold for the two conditions. 
decreased the flash-lag effect in active observation with that relationship.

Introspective reports from the observers were very similar to those in Experiment 1. That is, all of the observers reported that the automatic condition was easier than the manual condition, and they predicted that their performance was the most accurate in the automatic condition. All of them reported that, within the first several trials in the manual condition, they felt that they had moved the visual stimulus by the use of the computer mouse, and that there was no difficulty in controlling the stimulus movement and in judging the relative position of the flash and the moving stimulus.

\section{GENERAL DISCUSSION}

The results of the present experiments show that reduction of the flash-lag effect by active observation depends on the type of manual manipulation. We found a reduction of the flash-lag effect when the observers used the whole hand to manipulate the computer mouse to control the stimulus movement, regardless of the visibility of the observers' hand (Experiments 1 and 2), but we did not find such a reduction when the observers continuously pressed a key (Experiment 3) or manipulated a trackball (Experiment 4) to move the stimulus. Even when the observer manipulated the mouse to control the stimulus, the reduction of the flash-lag effect was restricted to a specific directional relationship between the stimulus movement and the hand movement. We found a reduction of the flash-lag effect when the upward (or downward) stimulus movement was coupled with the mouse movement away from (or toward) the observer, but we did not find a consistent reduction of the flash-lag effect for the opposite directional relationships between stimulus movement and hand movement, with which the observers were unfamiliar (Experiments 1, 2, and 5).

López-Moliner and Linares (2006) reported that the flash-lag effect was reduced when the flash was presented just after the observer's keypress. They proposed that this reduction of the flash-lag effect depends on the observer's prediction concerning the consequence of the keypress. Stork and Müsseler (2004) reported that the mislocalization of the vanishing point for a moving stimulus (representational momentum) was reduced when observers pursued the stimulus with their eyes and determined the vanishing point by pressing a key. They proposed that the predictability of the vanishing point enables eye movement to stop faster and to attain a greater spatial accuracy when an observer determines the vanishing point. In addition, Brown, Wilson, Goodale, and Gribble (2007) reported that learning in a motor system acquired under an artificial environmental force that was applied by a robotic manipulandum influences visual perception about how objects move in that artificial environment. They proposed that force information acquired by the motor system can contribute to how the motion of objects in the environment is predicted. The results of these studies suggest that, when the observer controlled the stimulus, the predictability of the moving stimulus improved visual per- ception in active observation. However, the predictability of the flash cannot explain the reduction of the flash-lag effect in the present consistent-motion condition and in the active condition in our previous study (Ichikawa \& Masakura, 2006a), because the timing and position of the flash randomly varied in all of these conditions; therefore, it was difficult to predict the timing and position of the flash. In addition, it is not plausible for one to assume a difference in the predictability of the flash between the consistent-motion and inconsistent-motion conditions in Experiments 1 and 2. We found no reduction in the flashlag effect in terms of active observation in Experiment 3, in which observers could most easily make a prediction because the stimulus moved with a constant velocity during the continuous keypress. These results indicate that, although the predictability of the flash and moving stimulus could affect the flash-lag effect (López-Moliner \& Linares, 2006) as well as the localization of the moving stimulus (Brown et al., 2007; Stork \& Müsseler, 2004), the reduction of the flash-lag effect in terms of active observation, which we have examined in our present study, does not depend only on the predictability of the flash, but also on the factors that are concerned with the manual manipulation.

In our previous study (Ichikawa \& Masakura, 2006a), we found that, even when the stimulus moved automatically, the flash-lag effect was reduced if the observer felt that he or she controlled the stimulus movement by a mouse. In addition, as we saw in the introduction, we found that the active observation reduced the RTs not only for the flash, but also for the shape change of the moving stimulus in that study. Therefore, we proposed that the observer's subjective mental set that he or she moves the stimulus facilitates visual processing for the whole visual field (not only for the moving stimulus manipulated by active mouse control) and, consequently, that it reduces the illusory flash-lag effect. In our present study, however, we found no such reduction of the flash-lag effect for active observation with the continuous keypress (Experiment 3) and with manipulating the trackball (Experiment 4), even when the observers felt that they had moved the stimulus by the use of those devices. These results suggest that the observer's subjective mental set that he or she moved the stimulus did not facilitate the visual processing by itself and that the reduction of the flash-lag effect in terms of active observation was restricted to a specific relationship between stimulus movement and hand movement.

There is a possibility that the size of the hand movement has an important role for the reduction of the flashlag effect in active observation. The hand movement in Experiment 3 was very small. In addition, the size of the hand movement in Experiment 4 was about half of that in Experiments 1 and 2. It is plausible that the proprioceptive signal from the hand has to exceed a specific level to affect the visual processing. However, even though the size of the hand movement was the same for both the consistent-motion and inconsistent-motion conditions, the reduction of the flash-lag effect was found only for the consistent-motion condition in Experiments 1 and 2. The results of these experiments indicate that the size of 
the hand movement is not the determinant of the reduction of the flash-lag effect in active observation.

What is the determinant for the reduction of the flashlag effect in terms of observers' active movements? In Experiments 1 and 2, we found no reduction of the flash-lag effect in the inconsistent-motion condition, whereas there was significant reduction in the consistent-motion condition. Because, in the observers' introspection, there was no subjective difference in controlling the stimulus movement between the consistent-motion and inconsistentmotion conditions, it was surprising that the reduction of the flash-lag effect was observed only in the consistentmotion condition. The results of Experiment 2 indicate that the visibility of the hand was not necessary for the reduction of the flash-lag effect in the consistent-motion condition. In Experiment 5, before the training sessions, we found no significant reduction of the flash-lag effect in the manual condition, in which the relationship between stimulus movement and mouse movement was the same as the one used for the inconsistent-motion condition in Experiments 1 and 2. These results imply that a specific directional relationship between a proprioceptive signal about observers' active hand movement in controlling the stimulus and a visual signal about stimulus movement is required for the reduction of the flash-lag effect.

In the manipulation of the computer mouse with the popular computer operating systems, the combination of the upward/downward movement of the stimulus on the display and the forward/backward movement of the computer mouse is more arbitrary than the combination of the leftward/rightward movement of the stimulus on the display and the leftward/rightward movement of the computer mouse. This arbitrariness may impair perceptual information processing and consequently reduce the stimulus-response compatibility effect (Phillips, Triggs, \& Meehan, 2005). This directional arbitrariness in combining the movements of devices and stimuli would exist not only for using a computer mouse, but also for using a keyboard and trackball. One of the anonymous reviewers pointed out that, in using a trackball, an observer would acquire proprioceptive information about hand motion that has the same direction as that for using a mouse, whereas they would acquire tactile information from the fingers and palm that is opposite of the proprioceptive information. This implies that the combination of directional information in using a trackball increased arbitrariness. It is plausible that this increased arbitrariness in using a trackball would impair the facilitating effect of the active observation on visual processing; therefore, it would prohibit the reduction of the flash-lag effect in Experiment 4.

Among the devices that were used for controlling stimulus movement in our present study, observers were most familiar with the computer mouse. That is, all of the observers had at least 4 years of experience in using their own personal computers with a mouse, although they had not trained to manipulate a cursor by the use of a keyboard or trackball. The finding that the reduction of the flash-lag effect was restricted to the consistent-motion condition in Experiments 1 and 2 suggests that the reduction depends on the experience of the observer. That is, regardless of the intrinsic arbitrariness in the directional relationship between the stimulus on the display and the computer mouse, the upward (or downward) movement of the stimulus on a display and the combination of the forward (or backward) movement of the mouse on a desk would be learned during the everyday use of personal computers. This directional relationship would be regarded as being less arbitrary after the repetitive use of personal computers. In fact, in Experiment 5, we found that training with the unfamiliar relationship between stimulus movement and hand movement could decrease the flash-lag effect, although the training was not enough to reduce the flashlag effect to a level that was smaller than that obtained in the automatic condition during the experiment. The learned relationship between the stimulus movement and the device movement (and, therefore, the observer's hand movement) would seem to facilitate visual processing and, consequently, reduce the illusory flash-lag effect in terms of active observation. Such a learning of the relationship between the stimulus movement and the hand or finger movement would not have occurred for pressing a key and rolling the trackball, because those manipulations were unfamiliar and arbitrary in controlling the stimulus movement on a computer display for our observers. This lack of learning would prohibit reduction of the flash-lag effect when the observer pressed a key (Experiment 3 ) or rolled the trackball (Experiment 4) to control the stimulus movement. For manipulating the keyboard or trackball to move the stimulus, however, we may expect that active observation with those devices will reduce the flash-lag effect if the repetitive trainings decrease the arbitrariness in the relationship between the stimulus and hand movements in using those devices.

In the present study, there was a discrepancy between the observers' introspective reports and performance. That is, in the introspective reports, all of the observers guessed that their performance would be more accurate in the automatic condition than in the consistent-motion and inconsistent-motion conditions in Experiments 1 and 2, although the most accurate condition was the consistentmotion condition. We found a similar discrepancy for the automatic and manual conditions in our previous study (Ichikawa \& Masakura, 2006a). In addition, in the present study, observers guessed that their performance would be more accurate in the automatic condition than in the consistent-motion and inconsistent-motion conditions in Experiment 4, although there was no consistent difference in the flash-lag effect among these conditions. In these experiments, observers had to pay attention not only to the visual stimuli, but also to their own hand motion in the consistent-motion and inconsistent-motion conditions, whereas they could focus on the stimuli in the automatic condition. Previous studies have found that taking away attention from the visual stimuli enhances the flash-lag effect (Baldo et al., 2002; Murakami, 2001; Shioiri et al., 2002). As was mentioned in the introduction, however, the results of our previous study (Ichikawa \& Masakura, 2006a) showed that active observation can facilitate processing all over the visual field. This result, as well as the 
present result that the flash-lag effect was reduced only in the consistent-motion condition with mouse manipulation, indicates that the familiar combination of visual stimulus movement and hand movement may cause facilitation of visual processing, regardless of the cognitive load caused by the division of attention to visual motion and hand motion. Facilitation of visual processing, which is obtained through everyday learning about the relationship between hand and stimulus movements, would raise the accuracy of visual processing and, consequently, reduce the visual illusion.

In previous studies, it has been found that activity in observation may facilitate visual processing not only for the temporal aspect (Ichikawa \& Masakura, 2006a), but also for the spatial aspect, such as depth perception from motion parallax (Rogers \& Graham, 1979), perceptual learning of the spatial structure of objects (Harman, Humphrey, \& Goodale, 1999; James, Humphrey, \& Goodale, 2001; Wang \& Simons, 1999), perceptual learning of the spatial relationships between visual information and proprioceptive body representations (Maravita, Clarke, Husain, \& Driver, 2002; Maravita, Husain, Clarke, \& Driver, 2001; Maravita, Spence, Kennett, \& Driver, 2002), and prismatic adaptation (Held, 1965; Welch, 1978; Welch, Widawski, Harrington, \& Warren, 1979). The other studies demonstrated that the proprioceptive signal of the hand affected the visual perception of the stimuli, which were attached to the moving hand (Carey \& Allan, 1996; DiZio, Lathan, \& Lackner, 1993). The results of these studies suggest that the observer's activity may contribute to the visual processing for different perceptual attributes. In particular, several studies have reported that observers' activities would facilitate visual motion processing. For instance, Spillmann, Pinna, Stürzel, and Werner (2003) reported that active head movement, which causes a change of viewpoint and, therefore, retinal position shift of the stimulus, facilitates the detection of an illusory motion signal. Wexler and Klam (2001) found that the active movement signal of an observer's hand and eyes contributed to the perception of trajectories of a moving stimulus. Blake, Sobel, and James (2004) reported that active touch of a 3-D object would disambiguate the perception of the 3-D structure from motion. Moreover, tactile motion involving the hand would activate the human MT+ (Blake et al., 2004; Hagen et al., 2002), which would be crucially involved in visual motion processing. Recently, Matsumiya and Shioiri (2008) found that active hand movement, which caused the rotation of a radial grating stimulus below the hand, enhanced the duration of the motion aftereffect for the grating stimulus only if the direction of the hand movement was consistent with the direction of the visual motion. Their results indicate that the effect of active observation on the visual processing of motion is restricted to the case in which there is an unambiguous relationship between the observer's active movement and object movement. Their result is similar to the present result in Experiments 1 and 2, that active observation facilitates visual processing only if the forward (or backward) hand movement on a desk is coupled with the upward (or downward) stimulus movement on a front parallel display. In addition, in many of the aforementioned studies that found the contributions of active observation to visual processing in different perceptual attributes, the change in the retinal image of the stimulus was concomitant with the observer's action. The results of the present study, together with those of the previous studies, imply that the proprioceptive signal generated by the observers' active motor control may contribute to visual processing if the unambiguous relationship between the observer's action and the change in the visual stimulus has been acquired through the observer's experience.

\section{AUTHOR NOTE}

A preliminary report on the present research was presented at the annual meeting of the Asian Conference on Vision, Matsue, Japan, in July 2006. This research was partially supported by the Japan Society for Promotion of Science Grant 21530760 to M.I. The authors thank J. Wolfe, B. Gibson, and three anonymous reviewers for their helpful comments and suggestions on earlier versions of the manuscript. Correspondence concerning this article should be addressed to M. Ichikawa, Department of Psychology, Chiba University, 1-33 Yayoi, Inage, Chiba, 263-8522, Japan (e-mail: ichikawa@L.chiba-u.ac.jp).

\section{REFERENCES}

Bachmann, T., Luiga, I., Põder, E., \& Kalev, K. (2003). Perceptual acceleration of objects in stream: Evidence from flash-lag displays. Consciousness \& Cognition, 12, 279-297.

Baldo, M. V. C., Kihara, A. H., Namba, J., \& Klein, S. A. (2002). Evidence for an attentional component of the perceptual misalignment between moving and flashing stimuli. Perception, 31, 17-30.

Blake, R., Sobel, K. V., \& James, T. W. (2004). Neural synergy between kinetic vision and touch. Psychological Science, 15, 397-402.

Brenner, E., \& Smeets, J. B. J. (2000). Motion extrapolation is not responsible for the flash-lag effect. Vision Research, 40, 1645-1648.

Brown, L. E., Wilson, E. T., Goodale, M. A., \& Gribble, P. L. (2007). Motor force field learning influences visual processing of target motion. Journal of Neuroscience, 27, 9975-9983.

Carey, D. P., \& Allan, K. (1996). A motor signal and "visual" size perception. Experimental Brain Research, 110, 482-486.

DiZio, P., LATHAN, C. E., \& LaCKNER, J. R. (1993). The role of brachial muscle spindle signals in assignment of visual direction. Journal of Neurophysiology, 70, 1578-1584.

Eagleman, D. M., \& Sejnowski, T. J. (2000a). Motion integration and postdiction in visual awareness. Science, 287, 2036-2038.

Eagleman, D. M., \& Sejnowski, T. J. (2000b). Reply to Patel et al. Science, 290, 1051.

Finney, D. J. (1971). Probit analysis. Cambridge: Cambridge University Press.

Hagen, M. C., Franzén, O., McGlone, F., Essick, G., Dancer, C., \& PARdo, J. V. (2002). Tactile motion activates the human middle temporal/V5 (MT/V5) complex. European Journal of Neuroscience, 16, 957-964

Harman, K. L., Humphrey, G. K., \& Goodale, M. A. (1999). Active manual control of object views facilitates visual recognition. Current Biology, 9, 1315-1318.

HeLD, R. (1965). Plasticity in sensory-motor systems. Scientific American, 213, 84-94.

ICHIKAWA, M., \& MASAKURA, Y. (2004). The connection of visual stimulus with observer's voluntary motion affects the flash-lag effect [Abstract]. Journal of Vision, 4(8), 581a.

ICHIKAWA, M., \& MASAKURA, Y. (2006a). Manual control of the visual stimulus reduces the flash-lag effect. Vision Research, 46, 21922203.

IChiKaWA, M., \& Masakura, Y. (2006b). Temporal precision in visual perception and impression formation in active observation. In K. Miura (Ed.), Proceedings of the First International Workshop on Kansei (pp. 32-35). Fukuoka, Japan. 
James, K. H., Humphrey, G. K., \& Goodale, M. A. (2001). Manipulating and recognizing virtual objects: Where the action is. Canadian Journal of Experimental Psychology, 55, 111-120.

KrekelberG, B., \& LAPPE, M. (2000). A model of the perceived relative positions of moving objects based upon a slow averaging process. Vision Research, 40, 201-215.

Krekelberg, B., \& LApPe, M. (2001). Neuronal latencies and the position of moving objects. Trends in Neurosciences, 24, 335-339.

López-Moliner, J., \& Linares, D. (2006). The flash-lag effect is reduced when the flash is perceived as a sensory consequence of our action. Vision Research, 46, 2122-2129.

Maravita, A., Clarke, K., Husain, M., \& Driver, J. (2002). Active tool use with the contralesional hand can reduce cross-modal extinction of touch on that hand. Neurocase, 8, 411-416.

Maravita, A., Husain, M., Clarke, K., \& Driver, J. (2001). Reaching with a tool extends visual-tactile interactions into far space: Evidence from cross-modal extinction. Neuropsychologia, 39, 580-585.

Maravita, A., Spence, C., Kennett, S., \& Driver, J. (2002). Tool-use changes multimodal spatial interactions between vision and touch in normal humans. Cognition, 83, B25-B34.

Matsumiya, K., \& Shiolri, S. (2008). Haptic movements enhance visual motion aftereffect [Abstract]. Journal of Vision, 8(6), 172a.

MurAKAMI, I. (2001). The flash-lag effect as a spatiotemporal correlation structure. Journal of Vision, 1, 126-136.

NiJHAWAN, R. (1994). Motion extrapolation in catching. Nature, 370, 256-257.

NiJHAWAN, R. (2002). Neural delays, visual motion and the flash-lag effect. Trends in Cognitive Sciences, 6, 387-393.

NiJHAWAN, R., \& KirschFeld, K. (2003). Analogous mechanisms compensate for neural delays in the sensory and the motor pathways: Evidence from motor flash-lag. Current Biology, 13, 749-753.

Patel, S. S., Ogmen, H., Bedell, H. E., \& Sampath, V. (2000). Flashlag effect: Differential latency, not postdiction. Science, 290, 1051a.

Phillips, J. G., Triggs, T. J., \& Meehan, J. W. (2005). Forward/up directional incompatibilities during cursor placement within graphical user interfaces. Ergonomics, 48, 722-735.

Rogers, B., \& Graham, M. (1979). Motion parallax as an independent cue for depth perception. Perception, 8, 125-134.

Sheth, B. R., Nishawan, R., \& Shimojo, S. (2000). Changing objects lead briefly flashed ones. Nature Neuroscience, 3, 489-495.

Shiolri, S., Yamamoto, K., \& YagUChI, H. (2002). Effect of attention on flash lagging [Abstract]. Journal of Vision, 2(7), 27a.

Spillmann, L., Pinna, B., Stürzel, F., \& Werner, J. S. (2003). Extraretinal factors required for visual illusions [Abstract]. Journal of Vision, 3(9), 656a.

Stork, S., \& Müsseler, J. (2004). Perceived localizations and eye movements with action-generated and computer-generated vanishing points of moving stimuli. Visual Cognition, 11, 299-314.

Wang, R. F., \& Simons, D. J. (1999). Active and passive scene recognition across views. Cognition, 70, 191-210.

Welch, R. B. (1978). Perceptual modification: Adapting to altered sensory environments. New York: Academic Press.

Welch, R. B., Widawski, M. H., Harrington, J., \& Warren, D. H. (1979). An examination of the relationship between visual capture and prism adaptation. Perception \& Psychophysics, 25, 126-132.

WeXler, M., \& Klam, F. (2001). Movement prediction and movement production. Journal of Experimental Psychology: Human Perception \& Performance, 27, 48-64.

Whitney, D., \& MuraKami, I. (1998). Latency difference, not spatial extrapolation. Nature Neuroscience, 1, 656-657.

Whitney, D., Murakami, I., \& Cavanagh, P. (2000). Illusory spatial offset of a flash relative to a moving stimulus is caused by differential latencies for moving and flashed stimuli. Vision Research, 40, 137-149.

(Manuscript received September 17, 2008; revision accepted for publication January 10, 2010.) 